\title{
BALANCED AVERAGING OF BILINEAR SYSTEMS WITH APPLICATIONS TO STOCHASTIC CONTROL
}

\author{
CARSTEN HARTMANN, BORIS SCHÄFER-BUNG, AND ANASTASIA THÖNS-ZUEVA
}

\begin{abstract}
We study balanced model reduction for stable bilinear systems in the limit of partly vanishing Hankel singular values. We show that the dynamics can be split into a fast and a slow subspace and prove an averaging principle for the slow dynamics. We illustrate our method with an example from stochastic control (density evolution of a dragged Brownian particle) and discuss issues of structure preservation and positivity.
\end{abstract}

\section{INTRODUCTION}

Modelling of chemical, physical or biological phenomena often leads to highdimensional systems of differential equations, resulting, e.g., from semi-discretized partial differential equations. Examples involve stochastic control problems [43], dissipative quantum dynamics [48], or metabolic networks [41].

For linear systems, balanced model reduction going back to [50] provides a rational basis for various approximation techniques that include easily computable error bounds [30]; see also [70, 2] and the references given there. The general idea of balanced model reduction is to restrict the system to the subspace of easily controllable and observable states which can be determined by the Hankel singular values associated with the system. For bilinear systems, however, neither a comprehensive theory nor robust numerical algorithms for efficiently solving the corresponding generalized Lyapunov equations are available, at least not to the same extent as in the linear case (e.g., see $[38,16,69,1,19,7])$. This article contributes to some theoretical aspects where only little attention is given to numerical feasibility and efficiency; regarding numerical issues we refer to, e.g, [6, 17].

For a certain class of stable bilinear systems we derive balanced reduced-order models by studying the limit of vanishing Hankel singular values. We do so by means of a multiscale analysis of the balanced equations of motion which are shown to collapse to a dimension reduced system when some of the Hankel singular values go to zero; see, e.g., [35, 36] for a related approach or [22, 47, 59, 29] in which low-rank perturbative approximations of transfer functions of linear systems are sought. To the best of our knowledge our approach is new, and, although it is based on computing an appropriate balanced form of the system equations which certainly becomes infeasible if the system is extremely high-dimensional ( $n \sim 10^{6}$ or larger), we see it not merely as an alternative, but rather as an extension to existing projectionbased methods such as Krylov subspace [54, 4, 15, 45, 46, 12, 10] or interpolation (moment-matching) methods [9, 23, 67], and empirical POD [37, 44, 13, 58]; see also $[7,60]$. A common feature of these methods is that they identify a subspace which contains the "essential" part of the dynamics, and we suggest to combine the numerically cheap identification of Krylov or POD subspaces with singular perturbation methods that have certain advantages in terms of structure preservation. (Another option that is discussed in [7] is to use Krylov methods as a preconditioner

Date: March 5, 2013.

Key words and phrases. Bilinear systems, model order reduction, balanced truncation, averaging method, Hankel singular values, generalized Lyapunov equations, stochastic control. 
that reduces the dimension of the system before balancing.) For a critical discussion of Krylov methods regarding preservation of moments and stability, and numerical efficiency we refer to [21]. We should mention related ideas in the direction of model order reduction for nonlinear systems based on a singular value analysis of the associated nonlinear Hankel operator that have been developed in a series of articles (see, e.g., [61] or [26, 27] for two recent accounts); the nonlinear balancing method establishes a splitting of the dynamics into subspaces corresponding to small and large singular values of the Hankel operator, and a subsequent elimination of the states that correspond to small singular values. The latter is either done by restricting the nonlinear vector field to the relevant subspace (balanced truncation), or by imposing an algebraic constraint that arises from the assumption that the irrelevant degrees of freedom are stationary (balanced residualization). In contrast, in our approach that resembles the well-known averaging method (see, e.g., [53] and the references therein), the Hankel singular values analysis serves only to identify suitable small parameters so as to arrive at a decomposition of the system into slow and fast variables. We then prove that in the limit of the small singular values going to zero, the fast dynamics converge (weakly) to a stationary probability measure which after averaging the slow dynamics against the invariant measure yields a lower-dimensional differential equation for the slow variables. Related theoretical works that analyze convergence of the averaging method for control systems in terms of differential inclusions can be found in [28, 20, 31, 64, 3]; see also [42] for a survey of singular perturbation techniques in linear control and filtering.

The structure of the article is as follows: In Section 2 we briefly review controllability and observability for linear and bilinear systems. Section 3 states the averaging problem and contains the main result, Theorem 3.2; our result is new in that we prove uniform convergence of the system to a limiting equation rather than a differential inclusion and establish a link with model order reduction based on the analysis of Gramians and Hankel singular values. A numerical example from stochastic control is discussed in Section 4, along with the problem of structurepreservation and positivity. The article has two appendices. In the first, Appendix A, we record diverse results related to the existence of the Gramian matrices on which our analysis is based, in the second, Appendix B, we propose an alternative approach to compute the solutions of generalized Lyapunov equations as covariance matrices of Markov diffusion processes.

\section{BilineAR CONTROL SySTEMS}

We consider bilinear control systems of the form

$$
\begin{aligned}
& \dot{x}=A x+\sum_{k=1}^{m} N_{k} x u_{k}+B u, \quad x(0)=x_{0}, \\
& y=C x
\end{aligned}
$$

where $x \in \mathbb{R}^{n}$ is the state vector, $u=\left(u_{1}, \ldots u_{m}\right), u_{i}: \mathbb{R} \rightarrow \mathbb{R}$ is the control, and $y \in \mathbb{R}^{l}$ denotes the vector of outputs. The matrices $A, B, C$ and $N_{k} \in \mathbb{R}^{n \times n}$, $k=1, \ldots, m$ are of appropriate dimensions (e.g., for $y \in \mathbb{R}^{l}$, we have $C \in \mathbb{R}^{l \times n}$ ). We suppose for the moment that the matrix $A$ is Hurwitz, i.e., all eigenvalues of $A$ have strictly negative real part. (Additional assumptions will be stated below.)

We seek matrices $\bar{A}, \bar{N}_{k} \in \mathbb{R}^{d \times d}, \bar{B} \in \mathbb{R}^{d \times m}$ and $\bar{C} \in \mathbb{R}^{l \times d}$ with $d \ll n$ such that

$$
\begin{aligned}
& \dot{\zeta}=\bar{A} \zeta+\sum_{k=1}^{m} \bar{N}_{k} \zeta u_{k}+\bar{B} u, \quad \zeta(0)=\zeta_{0}, \\
& \bar{y}=\bar{C} \zeta
\end{aligned}
$$


has an input-output behaviour that is similar to (2.1). In other words, we seek a reduced-order model with the property that for any admissible control input $u$ (to be defined below), the error $|\bar{y}(t)-y(t)|$ in the output signal is uniformly bounded in $t \in[0, T]$ with $0<T<\infty$.

2.1. Paradigm: linear systems. Our model reduction approach is based on an analysis of the Hankel singular values associated with the bilinear system (2.1). In doing so we borrow ideas from linear systems theory that relates the input-output properties of a stable system of equations (cf. [26, 27])

$$
\begin{aligned}
& \dot{x}=A x+B u, \quad x(0)=x_{0}, \\
& y=C x
\end{aligned}
$$

with the solution of the Lyapunov equations

$$
A W_{c}+W_{c} A^{*}+B B^{*}=0, \quad A^{*} W_{o}+W_{o} A+C^{*} C=0 .
$$

The positive semidefinite Gramian matrices $W_{c}$ and $W_{o}$ have suggestive physical interpretations: The controllability Gramian $W_{c}$ measures the control effort that is needed to drive the system to a state $x$; given two states $x_{1}, x_{2} \in \mathbb{R}^{n}$ with $\left|x_{1}\right|=\left|x_{2}\right|$, then $x_{1}$ can be reached with less control energy than $x_{2}$ if $x_{1}^{*} W_{c} x_{1}>x_{2}^{*} W_{c} x_{2}$. In particular, if $W_{c} x_{2}=0$, then the state $x_{2}$ cannot be reached at all, regardless of how strong the control field is; hence, it cannot contribute to the input-output behaviour or transfer function of the system. More precisely, we have (e.g., see [50, 61])

$$
x^{*} W_{c}^{-1} x=\min _{u \in U^{-}}\left\{\int_{-\infty}^{0}|u(t)|^{2} d t: x(0)=x, x(-\infty)=0\right\},
$$

where the minimum is over

$$
U^{-}=\mathcal{C}((-\infty, 0]) \cap L^{2}((-\infty, 0]),
$$

i.e., the completion of the continuous functions with respect to the $L^{2}$-norm. That is, $x^{*} W_{c}^{-1} x$ is the minimum energy in the $L^{2}$ sense that is needed to steer the system from the origin at time $t=-\infty$ to the state $x$ at $t=0$, assuming that the admissible controls are continuous, where we declare that the right hand side of (2.4) is infinite if a state $x \in \mathbb{R}^{n}$ cannot be reached by any $u \in U^{-}$. Conversely, the observability Gramian $W_{o}$ measures how much output energy can at least be extracted from the system when the input is bounded in $L^{2}$. More precisely,

$$
x^{*} W_{c} x \leq \max _{u \in U_{\alpha}^{+}}\left\{\int_{0}^{\infty}|y(t)|^{2} d t: x(0)=x, x(\infty)=0\right\} .
$$

where the maximum goes over the set

$$
U_{\alpha}^{+}=\left\{u \in \mathcal{C}([0, \infty)) \cap L^{2}([0, \infty)):\|u\|_{L^{2}([0, \infty))} \leq \alpha\right\}
$$

for any non-negative value of $\alpha$. Equality holds when $\alpha=0$, i.e., when the systems evolves freely with $u=0$. In particular, $W_{o} x=0$ means that no output signal can be extracted; the state is then called unobservable and does not contribute to the transfer function [50].

If (2.3) has neither unobservable nor uncontrollable states, then both $W_{c}$ and $W_{o}$ are positive definite. In this case one expects that states that are hardly controllable or observable will not play a major role for the transfer function. To make this precise we consider a contragredient transformation $x \mapsto T^{-1} x$ that makes the two Gramians equal and diagonal, i.e.,

$$
T^{-1} W_{c}\left(T^{-1}\right)^{*}=T^{*} W_{o} T=\Sigma,
$$

so that states that are least influenced by the input also have the least influence on the output and therefore can be neglected. Here $\Sigma=\operatorname{diag}\left(\sigma_{1}, \ldots, \sigma_{n}\right)>0$ is the diagonal matrix of Hankel singular values (HSVs). The HSVs are independent of 
the choice of coordinates as can be readily seen by noting that they are the square roots of the eigenvalues of $W_{c} W_{o}$, i.e.,

$$
T^{-1} W_{c} W_{o} T=\Sigma^{2} .
$$

It has been proved by Glover [30] that the following error bound holds for the rank $d$ approximant $\bar{y}$, that is obtained by projecting the coefficient matrices $A, B, C$ in (2.3) onto the space spanned by the first $d$ singular vectors:

$$
\sigma_{d+1}<\sup _{u \in L^{2}([0, \infty))} \frac{\|y-\bar{y}\|}{\|u\|} \leq 2\left(\sigma_{d+1}+\ldots+\sigma_{n}\right), \quad u \neq 0 .
$$

2.2. Realization theory for bilinear systems. For the bilinear system (2.1) we can define the controllability and the observability Gramian $W_{c}$ and $W_{o}$ as the solutions to the generalized Lyapunov equations

$$
A W_{c}+W_{c} A^{*}+\sum_{k=1}^{m} N_{k} W_{c} N_{k}^{*}+B B^{*}=0
$$

and

$$
A^{*} W_{o}+W_{o} A+\sum_{k=1}^{m} N_{k}^{*} W_{o} N_{k}+C^{*} C=0 .
$$

In contrast to the linear case, the generalized Gramians do not admit a straightforward energy interpretation in terms of controllability and observability functions; for a discussion of possible issues see [7, 14, 32]. Although equations (2.3)-(2.5) can be obtained by linearizing (2.1) about the stable fixed point $x=0$, such a linearization may not be very informative, for the bilinear term involving the control $u$ can grow without bound. The semidefinite generalized Gramians are useful in that their nullspace contains only uncontrollable and unobservable states $[38,16]$. As a consequence we can eliminate states from $\operatorname{ker}\left(W_{c}\right)$ and $\operatorname{ker}\left(W_{o}\right)$ without affecting the input-output behaviour of (2.1). Based on the linear system paradigm, we will further use the heuristic that states $x \in \mathbb{R}^{n}$ with $|x|=1$ for which $x^{*} W_{c} x$ or $x^{*} W_{o} x$ are small, are in some sense "negligible"; cf. [1, 7]. We will provide numerical evidence indicating that states corresponding to small HSVs of the generalized Gramians can indeed be neglected.

Remark 2.1. For bilinear systems the controllability function in (2.4) can be computed as the solution of a Hamilton-Jacobi-Bellman equation (see, e.g., [61]). However this nonlinear partial differential equation is a) not very handy for highdimensional problems, and b) we cannot expect it to have a classical (i.e., $\mathcal{C}^{1}$ ) solution at all. We further emphasize that the choice of the controllability and the observability function is not unique, and neither is their approximation by quadratic forms; see, e.g., [32, 33], or [34, 7] for a discussion of possible issues.

\section{BALANCED MODEL REDUCTION FOR BILINEAR SYSTEMS}

Let us come back to our bilinear system (2.1). We assume that the generalized Gramians $W_{c}, W_{o}$ are both positive definite and consider a balancing transformation $x \mapsto T^{-1} x$ under which the Gramians transform according to

$$
T^{-1} W_{\mathrm{c}}\left(T^{-1}\right)^{*}=\left(\begin{array}{cc}
\Sigma_{1} & 0 \\
0 & \Sigma_{2}
\end{array}\right)=T^{*} W_{\mathrm{o}} T .
$$

As in the linear case, the HSVs $\sigma_{i}$, i.e., the diagonal entries of $\Sigma=\left(\Sigma_{1}, \Sigma_{2}\right)$, are the square roots of the eigenvalues of the product $W_{c} W_{o}$. Hence they are independent of the choice of coordinates. Suppose that $\Sigma_{2} \ll \Sigma_{1}$ in the sense that the smallest 
entry of $\Sigma_{1}$ is much larger than the largest entry of $\Sigma_{2}$. For the reasons outlined in the last section, we may assume that in the balanced representation

$$
\left(A, B, N_{k}, C\right) \mapsto\left(T^{-1} A T, T^{-1} B, T^{-1} N_{k} T, C T\right), \quad k=1, \ldots, m,
$$

we can neglect all states corresponding to the almost invariant subspace of the small singular values $\Sigma_{2}$ without changing the input-output behaviour of (2.1) too much.

Small parameters. Starting from a balanced representation, we will now derive a dimension-reduced version of (2.1). Because of the positive definiteness we can decompose the two Gramians according to

$$
W_{c}=X X^{*}, \quad W_{o}=Y Y^{*}
$$

and do a singular value decomposition of the full-rank matrix $Y^{*} X$, i.e.,

$$
Y^{*} X=U \Sigma V^{*}=\left(\begin{array}{cc}
U_{1} & U_{2}
\end{array}\right)\left(\begin{array}{cc}
\Sigma_{1} & \mathbf{0} \\
\mathbf{0} & \Sigma_{2}
\end{array}\right)\left(\begin{array}{c}
V_{1}^{*} \\
V_{2}^{*}
\end{array}\right) .
$$

The partitioning $\Sigma_{1}=\operatorname{diag}\left(\sigma_{1}, \ldots, \sigma_{d}\right)$ and $\Sigma_{2}=\operatorname{diag}\left(\sigma_{d+1}, \ldots, \sigma_{n}\right)$ indicates which singular values are important and which are negligible. The remaining matrices satisfy $U_{1}^{*} U_{1}=V_{1}^{*} V_{1}=I_{d \times d}$ and $U_{2}^{*} U_{2}=V_{2}^{*} V_{2}=I_{r \times r}$ with $r=n-d$. In terms of the SVD, the balancing transformation $T$ and its inverse $S=T^{-1}$ read

$$
T=X V \Sigma^{-1 / 2}, \quad S=\Sigma^{-1 / 2} U^{*} Y^{*}
$$

as can be readily verified.

Now suppose $\sigma_{d+1} \ll \sigma_{d}$. As HSVs are coordinate invariant, the $\sigma_{d+1}, \ldots, \sigma_{n}>0$ can serve as dimensionless small parameters. Replacing $\Sigma_{2}$ by $\epsilon \Sigma_{2}$ in equation (3.2) and changing coordinates according to $x \mapsto S(\epsilon) x$ tells us where the small parameters $\Sigma_{2}$ enter the equations. Partitioning the thus obtained balancing matrices accordingly, then yields (see [35])

$$
S(\epsilon)=\left(\begin{array}{cc}
S_{11} & S_{12} \\
\epsilon^{-1 / 2} S_{21} & \epsilon^{-1 / 2} S_{22}
\end{array}\right), \quad T(\epsilon)=\left(\begin{array}{cc}
T_{11} & \epsilon^{-1 / 2} T_{12} \\
T_{21} & \epsilon^{-1 / 2} T_{22}
\end{array}\right)
$$

which gives rise to the balanced coefficients $\tilde{A}(\epsilon)=S(\epsilon) A T(\epsilon), \tilde{N}_{k}(\epsilon)=S(\epsilon) N_{k} T(\epsilon)$, $\tilde{B}(\epsilon)=S(\epsilon) B$, and $\tilde{C}(\epsilon)=C T(\epsilon)$. Specifically, we have

$$
\tilde{A}(\epsilon)=\left(\begin{array}{cc}
\tilde{A}_{11} & \epsilon^{-1 / 2} \tilde{A}_{12} \\
\epsilon^{-1 / 2} \tilde{A}_{21} & \epsilon^{-1} \tilde{A}_{22}
\end{array}\right)
$$

and similar expressions for $\tilde{N}(\epsilon), \tilde{B}(\epsilon)$, and $\tilde{C}(\epsilon)$ where the partitioning of the matrices is according to the splitting into large and small HSVs. In the last equation, $\tilde{A}=S(1) A T(1)$ denotes the balanced matrix $A$ for $\epsilon=1$, and we have used that the balancing transformations can be recast as $S(\epsilon)=\Gamma(\epsilon) S(1)$ and $T(\epsilon)=T(1) \Gamma(\epsilon)$ with $\Gamma(\epsilon)$ being the diagonal scaling matrix

$$
\Gamma(\epsilon)=\left(\begin{array}{cc}
I & 0 \\
0 & \epsilon^{-1 / 2} I
\end{array}\right) .
$$

In what follows, we shall omit the tilde on balanced matrices. In terms of the balanced variables $z=S(\epsilon) x$ with $z=\left(z_{1}, z_{2}\right)$ our bilinear system (2.1) turns into the singularly perturbed system of equations

$$
\begin{aligned}
\dot{z}_{1} & =A_{11} z_{1}+\frac{1}{\sqrt{\epsilon}} A_{12} z_{2}+\left(N_{11} z_{1}+\frac{1}{\sqrt{\epsilon}} N_{12} z_{2}+B_{1}\right) u \\
\sqrt{\epsilon} \dot{z}_{2} & =A_{21} z_{1}+\frac{1}{\sqrt{\epsilon}} A_{22} z_{2}+\left(N_{21} z_{1}+\frac{1}{\sqrt{\epsilon}} N_{22} z_{2}+B_{2}\right) u \\
y & =C_{1} z_{1}+\frac{1}{\sqrt{\epsilon}} C_{2} z_{2}
\end{aligned}
$$


where without loss of generality we have set $m=1$ in order to avoid the need to sum over the bilinear terms. ${ }^{1}$

Remark 3.1. In general we cannot be sure that the system is completely controllable and observable, in which case the Gramians are only semidefinite and the balancing transformation results in

$$
T^{-1} W_{\mathrm{c}}\left(T^{-1}\right)^{*}=\left(\begin{array}{cccc}
\Sigma_{1} & 0 & 0 & 0 \\
0 & \Sigma_{2} & 0 & 0 \\
0 & 0 & 0 & 0 \\
0 & 0 & 0 & 0
\end{array}\right), \quad T^{*} W_{\mathrm{o}} T=\left(\begin{array}{cccc}
\Sigma_{1} & 0 & 0 & 0 \\
0 & 0 & 0 & 0 \\
0 & 0 & \Sigma_{3} & 0 \\
0 & 0 & 0 & 0
\end{array}\right)
$$

with $\Sigma_{1}, \Sigma_{2}, \Sigma_{3}$ invertible and positive definite [2]. To save the previous scaling argument we may resort to some kind of regularization approach and replace the zero HSVs by entries of order $\epsilon^{s}$ with $s>1$. This will introduce an additional scale in equation (3.3) that must be taken into account. For the sake of clarity of the presentation, however, we refrain from treating the problem in such generality and assume throughout that the system is completely controllable and observable.

3.1. An averaging principle for bilinear control systems. We want to study the limit $\epsilon \rightarrow 0$ of vanishing small HSVs. For this purpose it is convenient to introduce the scaled variables $z_{2} \mapsto \sqrt{\epsilon} z_{2}$ by which (3.3) becomes

$$
\begin{aligned}
\dot{z}_{1} & =A_{11} z_{1}+A_{12} z_{2}+\left(N_{11} z_{1}+N_{12} z_{2}+B_{1}\right) u \\
\epsilon \dot{z}_{2} & =A_{21} z_{1}+A_{22} z_{2}+\left(N_{21} z_{1}+N_{22} z_{2}+B_{2}\right) u \\
y & =C_{1} z_{1}+C_{2} z_{2},
\end{aligned}
$$

where we remind the reader that the submatrices $A_{11}, A_{12}, \ldots$ are in balanced form. Equation (3.4) is an instance of a slow-fast system with $z_{1}$ being the slow variable and $z_{2}$ being fast. By rescaling time in the equations according to $t \mapsto t / \epsilon$ and assuming that $u$ can be bounded in some norm, we obtain the associated system

$$
\begin{aligned}
\dot{z}_{1} & =\mathcal{O}(\epsilon) \\
\dot{z}_{2} & =A_{21} z_{1}+A_{22} z_{2}+\left(N_{21} z_{1}+N_{22} z_{2}+B_{2}\right) u \\
y & =C_{1} z_{1}+C_{2} z_{2},
\end{aligned}
$$

that describes the dynamics on time scales of order $\epsilon$. Under suitable assumptions on the stability of the system and further assumptions on the controls that will be specified below it is reasonable to expect that, whenever $\epsilon$ is sufficiently small, the fast dynamics relax to a steady state or stationary distribution while the slow variable is effectively frozen. The general idea of the averaging principle then is to replace the fast variables in the slow equations by their stationary distributions, taking into account only their effective influence on the slow dynamics.

Here the situation is slightly different from averaging of uncontrolled (deterministic or stochastic) systems (see, e.g., [11, 25]), because the stationary distribution of the fast variables may depend on the controls, giving rise to averaged equations with measure-valued right hand side [28, 31, 64]. As we will argue below, however, the use of the balancing method entails certain restrictions on the admissible controls $u$ that allow for proving stronger results, namely, uniform convergence to a controlled differential equation governing the slow dynamics.

\footnotetext{
${ }^{1}$ This choice is merely conventional - neither the balancing procedure, including the solution of the generalized Lyapunov equations, nor the singular perturbation analysis rely on fact that the input is scalar. All results can be easily extended to the case of multiple inputs.
} 
Statement of the main result. The following assumptions on the system matrices and the controls will be used throughout:

Assumption 1: The system (3.4) is BIBO stable, i.e., for all $s \in[-c, c]$ and some $c>0$, the eigenvalues of the matrix $A+s N$ have strictly negative real part.

Assumption 2: The matrix $A_{22}$ in (3.4) is Hurwitz; its eigenvalues $\lambda_{i}$ satisfy

$$
\max _{i} \Re\left(\lambda_{i}\left(A_{22}\right)\right) \leq-\delta<0 .
$$

Assumption 3: The controls $u:[0, \infty) \rightarrow E \subset \mathbb{R}$ are bounded continuous functions with finite energy, i.e., $u \in \mathcal{C}_{b}([0, \infty)) \cap L^{2}([0, \infty))$.

Assumption 4: The controls $u=u^{\epsilon}$ are from the class of relatively slow controls in the sense of Gaitsgory [28], i.e., $u^{\epsilon}(t)=u\left(t / \epsilon^{\gamma}\right)$ with some $0<\gamma<1$.

The fourth assumption, which will play a role in the convergence proof below, states that the controls act on an intermediate time scale between the slow and the fast variables, the third assumption, that is common in balanced model reduction for linear systems, implies that the control decays asymptotically as $t \rightarrow \infty$, which guarantees that the fast dynamics relax to a stationary distribution that is independent of $u$. Indeed, if, in equation (3.5), we freeze the slow variable at $z_{1}=\zeta$, the fast dynamics are obtained as the solution of

$$
\dot{z}_{2}=A_{21} \zeta+A_{22} z_{2}+\left(N_{21} \zeta+N_{22} z_{2}+B_{2}\right) u,
$$

If we then let $\varphi_{\zeta}^{t, t_{0}}(\xi)$ denote the solution of the last equation with initial condition $z_{2}\left(t_{0}\right)=\xi$, it follows by Assumptions 2 and 3 that

$$
\lim _{t \rightarrow \infty} \varphi_{\zeta}^{t, t_{0}}\left(z_{2}\right)=-A_{22}^{-1} A_{21} \zeta
$$

which is equivalent to the statement that, the fast dynamics converge to a unique stationary distribution given by the Dirac measure concentrated at $-A_{22}^{-1} A_{21} \zeta$. The averaging principle for uncontrolled systems then suggests that in (3.4) we may replace $z_{2}(t)$ by its steady state $-A_{22}^{-1} A_{21} z_{1}(t)$ which yields a closed equation for $z_{1}$ and the output variable $y$. Our main result is the following averaging principle.

Theorem 3.2. Let $y_{\epsilon}(t)$ be the observed solution of (3.4), satisfying Assumption 1-4 above. Further let $\bar{y}(t)$ be the output of the reduced system

$$
\begin{aligned}
& \dot{\zeta}=\bar{A} \zeta(t)+\left(\bar{N} \zeta+B_{1}\right) u, \quad \zeta(0)=\zeta_{0}, \\
& \bar{y}=\bar{C} \zeta
\end{aligned}
$$

with the coefficients

$$
\bar{A}=A_{11}-A_{12} A_{22}^{-1} A_{21}, \bar{N}=N_{11}-N_{12} A_{22}^{-1} A_{21}, \bar{C}=C_{1}-C_{2} A_{22}^{-1} A_{21} .
$$

Then

$$
\lim _{\epsilon \rightarrow 0}\left|y_{\epsilon}(t)-\bar{y}(t)\right|=0
$$

uniformly for $t \in[0, T]$ and for all initial conditions $\left(z_{1}(0), z_{2}(0)\right)$ with $z_{1}(0)=\zeta_{0}$.

3.2. Proof of the averaging principle. For the proof of Theorem 3.2, it suffices to establish convergence of the state variables, ignoring the output. For the sake of convenience, we write the first two equations in (3.4) as the system of equations

$$
\begin{aligned}
\dot{z}_{1} & =f\left(z_{1}, z_{2}, u\right) \\
\epsilon \dot{z}_{2} & =g\left(z_{1}, z_{2}, u\right)
\end{aligned}
$$

with $\left(z_{1}, z_{2}\right) \in \mathbb{R}^{d} \times \mathbb{R}^{n-d}$ and $0<\epsilon \ll 1$ and $\left(z_{1}, z_{2}\right)=(0,0)$ being a global asymptotically stable fixed point of the control-free system (i.e., for $u=0$ ).

We prove that in the limit $\epsilon \rightarrow 0$ and for $u$ being admissible in the sense of Assumptions 3 and 4 on page 7 , the slow component $z_{1}$ converges uniformly to $\zeta$ that is governed by

$$
\dot{\zeta}=f(\zeta, m(\zeta), u)
$$


where $z_{2}=m(\zeta)$ is the graph representation of the limiting invariant subspace

$$
M=\left\{\left(\zeta, z_{2}\right) \in \mathbb{R}^{d} \times \mathbb{R}^{n-d}: g\left(\zeta, z_{2}, 0\right)=0\right\} \subset \mathbb{R}^{n}
$$

that the fast dynamics approach as $\epsilon \rightarrow 0$.

Contractivity and admissible controls. Suppose that $z_{1}=\zeta$ is fixed. By the assumption that $A_{22}$ is Hurwitz and the fact that $u$ decays as $t \rightarrow \infty$ the fast subsystem has a unique stable fixed point zero (recall that $u$ is continuous and square integrable). That is, for all $\zeta \in \mathbb{R}^{d}$ fixed, the solution $\varphi_{\zeta}^{t}(\xi):=\varphi_{\zeta}^{t, t_{0}}(\xi)$ of the associated system (here and in the following we set $t_{0}=0$ )

$$
\dot{z}_{2}=g\left(\zeta, z_{2}, u\right), \quad z_{2}(0)=\xi
$$

has a unique exponentially attracting fixed point, i.e.,

$$
\lim _{t \rightarrow \infty} \varphi_{\zeta}^{t}(\xi)=m(\zeta)=-A_{22}^{-1} A_{21} \zeta
$$

uniformly in $\zeta$ and independently of the initial condition $z_{2}(0)=\xi$. More precisely, we observe that $g(\zeta, m(\zeta), 0)=0$ where $g$ meets the following contractivity condition: for any $\zeta \in \mathbb{R}^{d}$ and $z_{2}, \tilde{z}_{2} \in \mathbb{R}^{n-d}$ there exist $\alpha, \delta>0$ such that

$$
\left\langle g\left(\zeta, z_{2}, u\right)-g\left(\zeta, \tilde{z}_{2}, u\right), z_{2}-\tilde{z}_{2}\right\rangle \leq-\alpha\left|z_{2}-\tilde{z}_{2}\right|^{2} .
$$

for all admissible controls $u$ with $|u| \leq \delta$. The contractivity condition is met by the fact that the spectrum of $A_{22}$ is bounded away from the imaginary axis; as the controls $u$ are decaying, this means that there exists a $t^{*}>0$, such that $A_{22}+u N_{22}$ is Hurwitz for all $t \geq t^{*}$. This entails (3.8), which, by Gronwall's Lemma, gives the fixed-point property.

Convergence to the invariant subspace. In order to avoid the inflationary use of symbols, we implement the following notation: we call $L>0$ a uniform Lipschitz constant and let $U \times V \subset \mathbb{R}^{d} \times \mathbb{R}^{n-d}$ be an open set such that

$$
\begin{aligned}
\left|f\left(z_{1}, z_{2}, u\right)\right| \leq L & \forall\left(z_{1}, z_{2}\right) \in U \times V \\
\left|\nabla f\left(z_{1}, z_{2}, u\right)\right| \leq L & \forall\left(z_{1}, z_{2}\right) \in U \times V \\
\left|\nabla m\left(z_{1}\right)\right| \leq L & \forall z_{1} \in U .
\end{aligned}
$$

Existence of $L<\infty$ is guaranteed by the Assumptions 1-3 above. In particular we may choose $L$ such that

$$
\text { . }\left|g\left(z_{1}, m\left(z_{1}\right), u\right)\right| \leq L u \quad \forall\left(z_{1}, z_{2}\right) \in U \times V
$$

We now define the deviations of the fast variable from the invariant manifold by

$$
\eta=z_{2}-m(\zeta)
$$

As a first step we estimate the rate at which $\eta$ goes to zero as $\epsilon \rightarrow 0$. Since

$$
\dot{\eta}=\dot{z}_{2}-\nabla m(\zeta) \dot{\zeta}
$$

the augmented set of variables $\left(\zeta, \eta, z_{2}\right)$ is governed by the joint system of equations

$$
\begin{aligned}
\dot{\zeta} & =f(\zeta, m(\zeta)+\eta, u) \\
\dot{\eta} & =\frac{1}{\epsilon} g(\zeta, m(\zeta)+\eta, u)-\nabla m(\zeta) f(\zeta, m(\zeta), u) \\
\dot{z}_{2} & =\frac{1}{\epsilon} g(\zeta, m(\zeta)+\eta, u)
\end{aligned}
$$

that is equivalent to (3.7). By adding zero, the second equation can be recast as

$$
\dot{\eta}=\frac{1}{\epsilon}(g(\zeta, m(\zeta)+\eta, u)-g(\zeta, m(\zeta), u)+g(\zeta, m(\zeta), u))-\nabla m(\zeta) f(\zeta, m(\zeta), u) .
$$

Lipschitz continuity of $f, g$ and $m$ and the Cauchy-Schwarz inequality entail

$$
g(\zeta, m(\zeta), u) \leq L u, \quad\langle\nabla m(\zeta) f(\zeta, m(\zeta), u), \eta\rangle \leq L^{2}|\eta|
$$


Using further equation (3.8), i.e.,

$$
\langle g(\zeta, m(\zeta)+\eta, u)-g(\zeta, m(\zeta), u), \eta\rangle \leq-\alpha|\eta|^{2},
$$

we obtain the following differential inequality for $\eta$ :

$$
\begin{aligned}
\frac{1}{2} \frac{d}{d t}|\eta|^{2} & =\langle\eta, \dot{\eta}\rangle \\
& =\frac{1}{\epsilon}\langle g(\zeta, m(\zeta)+\eta, u), \eta\rangle-\langle\nabla m(\zeta) \bar{f}(\zeta), \eta\rangle \\
& \leq-\frac{\alpha}{\epsilon}|\eta|^{2}+M|\eta| .
\end{aligned}
$$

with $M=L^{2}+L u / \epsilon$. Completing the square yields

$$
\frac{1}{2}\left(\delta M-\frac{|\eta|}{\delta}\right)^{2}>0 \Rightarrow \frac{1}{2}\left(\delta^{2} M^{2}+\frac{|\eta|^{2}}{\delta^{2}}\right)>L|\eta|
$$

for any $\delta \in \mathbb{R}$. Setting $\delta=\sqrt{\epsilon / \alpha}$, we therefore find

$$
\begin{aligned}
\frac{1}{2} \frac{d}{d t}|\eta|^{2} & \leq-\frac{\alpha}{\epsilon}|\eta|^{2}+\frac{1}{2}\left(\delta^{2} M^{2}+\frac{|\eta|^{2}}{\delta^{2}}\right) \\
& \leq-\frac{\alpha}{2 \epsilon}|\eta|^{2}+\frac{\epsilon}{2 \alpha} M^{2} .
\end{aligned}
$$

Thus Gronwall's Lemma gives the bound

$$
|\eta|^{2} \leq \exp \left(-\frac{\alpha}{2 \epsilon} t\right)\left(|\eta(0)|^{2}+\frac{\epsilon}{2 \alpha} \int_{0}^{t} \exp \left(\frac{\alpha}{2 \epsilon} s\right) M_{s}^{2} d s\right)
$$

where the subscript $M_{s}$ indicates the time dependence of $M$ through $u$. If we set $\hat{u}=\max \left\{u\left(t / \epsilon^{\gamma}\right): t \in(0, T]\right\}$ the integral can be bounded from above by

$$
|\eta|^{2} \leq \exp \left(-\frac{\alpha}{2 \epsilon} t\right)|\eta(0)|^{2}+\frac{\epsilon^{2} \hat{M}^{2}}{\alpha^{2}}\left(1-\exp \left(-\frac{\alpha}{2 \epsilon} t\right)\right) .
$$

with $\epsilon^{2} \hat{M}^{2}=\epsilon^{2} L^{4}+2 \epsilon L^{3} \hat{u}+L^{2} \hat{u}^{2}$. Since $u\left(t / \epsilon^{\gamma}\right) \rightarrow 0$ for any fixed $t>0$ as $\epsilon \rightarrow 0$ we conclude that $\hat{u} \rightarrow 0$ and therefore

$$
\lim _{\epsilon \rightarrow 0}|\eta(t)|^{2}=0
$$

for all $t \in[0, T]$ which implies $\eta \rightarrow 0$. This completes the first part of the proof.

Convergence of solutions. In order to show that convergence of the fast dynamics to the invariant subspace implies uniform convergence $z_{1} \rightarrow \zeta$, we note that

$$
\dot{z}_{1}=f(\zeta, m(\zeta)+\eta, u)
$$

by definition of $\eta$, and

$$
\dot{\zeta}=f(\zeta, m(\zeta), u)
$$

Using Cauchy-Schwarz and Lipschitz continuity of $f$, it readily follows that

$$
\begin{aligned}
\frac{1}{2} \frac{d}{d t}\left|z_{1}-\zeta\right|^{2} & =\left\langle z_{1}-\zeta, f(\zeta, m(\zeta)+\eta, u)-f(\zeta, m(\zeta), u)\right\rangle \\
& \leq\left|z_{1}-\zeta\right||f(\zeta, m(\zeta)+\eta, u)-f(\zeta, m(\zeta), u)| \\
& \leq L\left|z_{1}-\zeta\right||\eta| .
\end{aligned}
$$

By completing the square we obtain the inhomogeneous differential inequality

$$
\frac{d}{d t}\left|z_{1}-\zeta\right|^{2} \leq L^{2}\left|z_{1}-\zeta\right|^{2}+|\eta|^{2}
$$

with $|\eta|^{2}$ as given by (3.9) above. For $z_{1}(0)=\zeta(0)$, Gronwall's Lemma gives

$$
\left|z_{1}-\zeta\right|^{2} \leq \int_{0}^{t} \exp (L(t-s))|\eta(s)|^{2} d s .
$$


The assertion that $z_{1} \rightarrow \zeta$ uniformly on $[0, T]$ follows upon inserting (3.9) in the last inequality and integrating, viz.,

$$
\left|z_{1}-\zeta\right|^{2} \leq C e^{L t}\left(\epsilon|\eta(0)|^{2}+\epsilon^{\gamma}\right)
$$

In equation (3.4), $y$ is a linear transformation of the state variables $z_{1}$ and $z_{2}=$ $\eta+m\left(z_{1}\right)$. Hence (3.10) implies convergence $y \rightarrow \bar{y}$ which proves Theorem 3.2.

Remark 3.3. We should mention a similar result that is due to Watbled [68]. The author proves uniform convergence of the slow process on the interval $[0, \infty)$ to the solutions of a differential inclusion. The proof relies on the construction of a suitable Lyapunov functional by which convergence of the fast dynamics to an invariant manifold can be shown. Although it does not give convergence rates for $\epsilon \rightarrow 0$, it proves that the error remains bounded for all times (given that certain technical conditions are met that are difficult to verify in practice). General convergence results for averaged control systems using differential inclusion techniques are due to Gaitsgory and co-workers [28, 31, 64].

Remark 3.4. Upon inspecting (3.10) we see that our error bound consists of two parts the first of which depends on the deviation of the initial condition $z_{2}(0)$ from the invariant subspace $m\left(z_{1}(0)\right)$. That is, the first term is due to the initial relaxation of the fast dynamics to the steady state, whereas the second term describes the actual approximation error that arises from replacing $f\left(z_{1}, z_{2}, u\right)$ by $f\left(z_{1}, m\left(z_{1}\right), u\right)$. Further notice that the upper bound for the error grows like $\exp (L t)$, i.e., for $t=\mathcal{O}(-\ln \epsilon)$ the upper bound becomes essentially of order 1 .

3.3. Comparison with balanced truncation and residualization. The reader may wonder how the averaging principle relates to the usual method of balanced truncation and other singular perturbation approaches (that are also known by the name of balanced residualization). Roughly speaking balanced truncation amounts to setting $z_{2}=0$ in the balanced equations (3.4), whereas singular perturbation methods seek a closure of the equations by arguing that $\dot{z}_{2} \approx 0$.

Clearly, our approach belongs to the second category as we use that $\dot{z}_{2} \rightarrow 0$ in the associated system (3.5). Note, however, that simply setting $\dot{z}_{2}=0$ as is stipulated by residualization methods (see, e.g., $[47,29,27]$ ) and solving the resulting algebraic equations for $z_{2}$ is different from letting $\dot{z}_{2} \rightarrow 0$; in fact when the time scales of the two subsystems are clearly separated, the point-wise condition $\dot{z}_{2}=0$ of the residualization may not be very meaningful, e.g., when the fast variables oscillate infinitely fast around the stationary mean value zero in which case $\dot{z_{2}} \neq 0$. However assuming a certain degree of "hyperbolicity" of the fast subsystem and suitable decay properties of the controls, it is possible to show (e.g., see [31]) that the fast variables weakly converge to an invariant measure on time scales of order one (e.g., a Gaussian with mean zero in the previous oscillatory scenario).

In the case of linear systems, both truncation and residualization can be shown to yield reduced systems that preserve stability and that obey the usual $H^{\infty}$ error bound [30]. Although we strongly believe that a similar result may hold for bilinear systems, it is clear that proving such a result would require completely different mathematical techniques, e.g., diffusive limits [40,52] or differential inclusion techniques [68] which is beyond the scope of this article.

Remark 3.5. It is interesting to note that the limiting equations (3.6) resemble the result of the Schur complement method that is employed for solving partial differential equations on complicated domains (see, e.g., [55]). In the language of our approach this is to say that we decompose our system's state space (i.e., the computational domain) into controllable/observable and hardly controllable/observable subspaces and restrict the solution to the first one where the latter enters the problem in form of stationary boundary terms. 


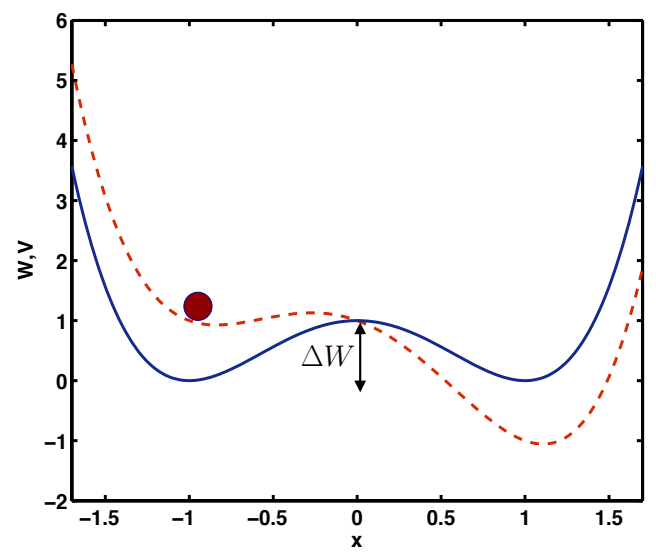

FIgURE 1. The optical tweezer acting with force $u$ tilts the original double well $W$ potential (solid line) to $V=W-u x$ (dashed line).

\section{Applications and nUmerical illustration}

We shall now illustrate the balanced model reduction approach with an example from stochastic control: a semi-discretized Fokker-Planck equation with external forcing. The Fokker-Planck or forward Kolmogorov equation (see [51]) describes the evolution of the probability distribution of the solution to a stochastic differential equation. By being probabilities, the state variables are nonnegative. Moreover, the system comes with a simple eigenvalue zero that corresponds to the stationary distribution of the system and which means that it cannot be BIBO stable. Whereas the latter problem can be easily dealt with by an appropriate shifting and scaling procedure that is described in Appendix A below, preservation of positivity turns out to be a more delicate issue and fails to hold in general.

4.1. Dragged Brownian particle. Consider a stochastic particle on the real line assuming states $x \in \mathbb{R}$ that is confined by a double-well potential

$$
W(x)=\left(x^{2}-1\right)^{2} .
$$

Suppose that initially the particle is in the left well and we want to drag it to the right well; even without external forcing the particle will eventually hop to the right well, but on a time scale that is of the order $\exp (\Delta W / \vartheta)$ where $\Delta W=W(0)$ denotes the energy barrier that the particle has to overcome and $\vartheta>0$ is the (dimensionless) temperature of the system [25]; in typical application scenarios, $\vartheta$ is small, so that the typical transition time (more precisely: the mean first exit time) is enormous; by dragging the particle over the barrier the transition rate can be considerably increased.

Situations of this kind arise, e.g., in atomic force microscopy $[56,49]$ or singlemolecule pulling experiments $[24,39]$ in which the system is typically manipulated by an optical tweezer. To lowest order, a reasonably good model for the interaction with the particle with an optical tweezer is

$$
\phi(x ; u)=-u x
$$

where $u$ denotes the force exerted on the particle (see Figure 1). The motion of the particle is then governed by the stochastic differential equation

$$
d X_{t}=-\nabla V\left(X_{t}, t\right) d t+\sqrt{2 \vartheta} d W_{t}, \quad X_{0} \text { in the left well, e.g., } X_{0}=-1,
$$


with $0<\vartheta \leq 1 / 2$ and $V(x, t)=W(x)+\phi\left(x, u_{t}\right)$. Equivalently, the dynamics of the particle can be described in terms of its probability distribution function

$$
\rho(x, t) d x=\mathbf{P}\left[X_{t} \in[x, x+d x)\right]
$$

that is governed by the Fokker-Planck equation

$$
\frac{\partial \rho}{\partial t}=\vartheta \Delta \rho+\nabla \cdot(\rho \nabla V), \quad \rho(x, 0)=\rho_{0}(x) .
$$

Here $\rho_{0} \neq 0$ denotes a smooth initial probability distribution for the diffusion process (4.1). For $u=0$ and for smooth potentials $W$ that grow at least quadratically (as in our case) all solutions converge to the Boltzmann distribution $\rho_{\infty} \propto$ $\exp (-W / \vartheta)$ that is the unique solution of the elliptic equation

$$
\vartheta \Delta \rho+\nabla \cdot(\rho \nabla V)=0
$$

where the rate of convergence is exponential in the first nonzero eigenvalue of the operator $L$ defined by the right hand side of (4.2), i.e., $L \rho=\vartheta \Delta \rho+\nabla \cdot(\rho \nabla V)$; see the seminal article [5] or the textbook [65] for details.

Discrete Fokker-Planck equation. Now let us discretize the parabolic partial differential equation (4.2) on a finite spatial domain $I \subset \mathbb{R}$, say, $I=[a, b]$. Conservation of probability then requires that the (outwards) probability flux

$$
J(\rho)=\vartheta \nabla \rho+\rho \nabla V
$$

vanishes at the boundaries $a$ and $b$, so that (4.2) assumes the form

$$
\begin{aligned}
\frac{\partial \rho}{\partial t}=\vartheta \Delta \rho+\nabla \cdot(\rho \nabla V) & (x, t) \in(a, b) \times(0, T] \\
0=\vartheta \nabla \rho+\rho \nabla V & (x, t) \in\{a, b\} \times[0, T] \\
\rho_{0}=\rho & (x, t) \in(a, b) \times\{0\} .
\end{aligned}
$$

As we are not interested in particularly sophisticated spatial discretization schemes, we choose the simplest finite difference scheme to illustrate the basic idea: given a grid $\left\{x_{1}=a, x_{2}=a+h, x_{3}=a+2 h, \ldots, x_{n}=b\right\}$ and defining $\rho_{i}(t)=\rho\left(x_{i}, t\right)$ the finite difference discretization of the initial boundary value problem (4.3) reads

$$
\dot{\rho}_{i}=\frac{\vartheta}{h^{2}}\left(\rho_{i+1}-2 \rho_{i}+\rho_{i-1}\right)+\frac{W^{\prime}\left(x_{i}\right)-u}{2 h}\left(\rho_{i+1}-\rho_{i-1}\right)+W^{\prime \prime}\left(x_{i}\right) \rho_{i} .
$$

for $i=2, \ldots, n-1$. At the boundaries $x_{1}=a$ and $x_{n}=b$ we have

$$
\dot{\rho}_{1}=\frac{2 \vartheta}{h^{2}}\left(\rho_{2}-\rho_{1}\right)+\left(\frac{2 W^{\prime}(a)}{h}-\frac{\left(W^{\prime}(a)\right)^{2}}{\vartheta}+W^{\prime \prime}(a)+\frac{u W^{\prime}(a)}{\vartheta}\right) \rho_{1}
$$

and

$$
\dot{\rho}_{n}=\frac{2 \vartheta}{h^{2}}\left(\rho_{n-1}-\rho_{n}\right)+\left(-\frac{2 W^{\prime}(b)}{h}-\frac{\left(W^{\prime}(b)\right)^{2}}{\vartheta}+W^{\prime \prime}(b)+\frac{u W^{\prime}(b)}{\vartheta}\right) \rho_{n} .
$$

The initial value is given by the vector with the nonnegative entries $\rho_{i}(0)=\rho_{0}\left(x_{i}\right)$. In matrix-vector notation, the last equations can be compactly written as

$$
\dot{\rho}=A \rho+N \rho u, \quad \rho(0)=\rho_{0},
$$

where we have ignored boundary terms that are quadratic in $u$. By our choice of a spatial discretization scheme, $-A \in \mathbb{R}^{n \times n}$ is an $M$-matrix with a simple eigenvalue 0 that corresponds to the discretized stationary distribution $\rho_{\infty} \propto \exp (-W / \vartheta)$ on $I \subset \mathbb{R}$. Since the diffusion process (4.1) is reversible, i.e., it satisfies detailed balance, it follows moreover that $A$ has only real eigenvalues (provided that the discretization is sufficiently fine); for some results on the spectral theory of the Fokker-Planck equations the reader is referred to the standard textbook [57]. 

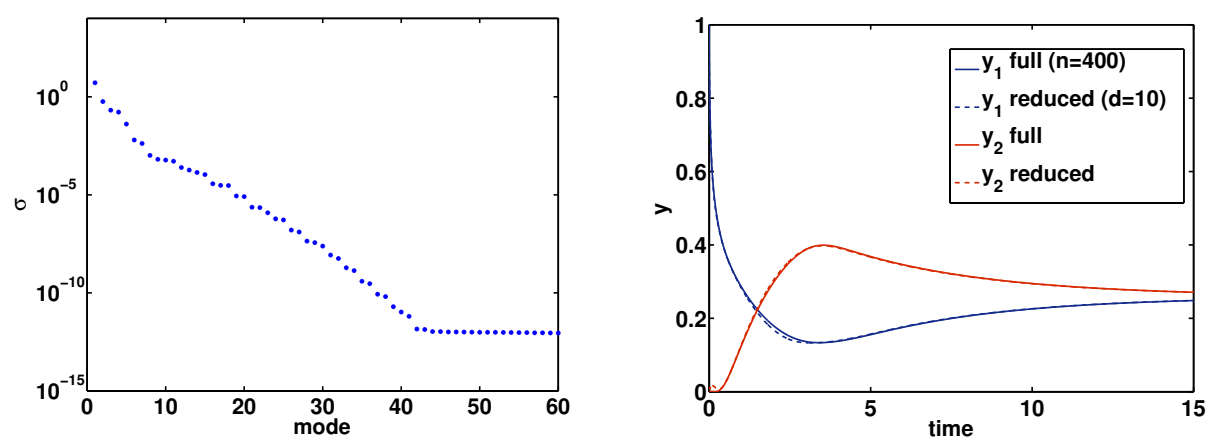

Figure 2. Controlled Fokker-Planck equation: the first 60 HSVs (left panel) and the approximant of degree $d=10$ (right panel). The blue curve shows the probability in the left well whereas the red curve shows how the right well is populated.

We augment (4.4) by an output equation. To this end, we introduce the observable $y=\left(y_{1}, y_{2}\right)$ with $y_{i} \geq 0$ denoting the probabilities to be in the left well or the right well which yields the homogeneous system

$$
\begin{aligned}
& \dot{\rho}=A \rho+N \rho u \\
& y=C \rho .
\end{aligned}
$$

The observation matrix $C \in \mathbb{R}^{2 \times n}$ is given by $C=\left(P_{L}, P_{R}\right)$ where $P_{L}, P_{R} \in \mathbb{R}^{n}$ denote the discrete characteristic functions of left and right potential well.

Clearly, in its present form, (4.5) cannot be controllable at all, for $B=0$ and therefore one cannot escape the the zero state $\rho=0$. Even though the zero state is certainly not of particular interest, notwithstanding that is is not a probability distribution, we can exploit the fact that the matrix $A$ has a nontrivial kernel $v=\rho_{\infty}$ that is given by the stationary distribution of the diffusion process (4.1): shifting the state variables according to $\rho \mapsto \rho+v$ will generate an additive term $B u=-N v u$ that makes the system controllable from the zero state; we refer to Section A below for the details (also regarding the violation of the stability requirement stated on page 7), and simply assume that the discrete Fokker-Planck equation (4.5) is already in balanced form, i.e., we assume that Gramians, HSVs and balancing transforms have been computed. In partitioned form (4.5) reads

$$
\begin{aligned}
\dot{\rho}_{1} & =A_{11} \rho_{1}+A_{12} \rho_{2}+\left(N_{11} \rho_{1}+N_{12} \rho_{2}\right) u \\
\epsilon \dot{\rho}_{2} & =A_{21} \rho_{1}+A_{22} \rho_{2}+\left(N_{21} \rho_{1}+N_{22} \rho_{2}\right) u \\
y & =C_{1} \rho_{1}+C_{2} \rho_{2},
\end{aligned}
$$

where we have inserted the scaling parameter $\epsilon>0$ to make the connection with the averaging principle, Theorem 3.2, clear.

Since the stationary distribution, i.e., the kernel of $A$ is easily controllable and observable, it is safe to assume that the weakly controllable and observable modes lie in the complementary subspace. In other words, we may assume that $A_{22}$ is Hurwitz (which will be checked numerically) so that the dominant subspace is asymptotically stable as we have assumed in the proof of Theorem 3.2. Then, as $\epsilon \rightarrow 0$, the dynamics converge to the solutions of the averaged system

$$
\begin{aligned}
\dot{\rho}_{1} & =\left(A_{11}-A_{12} A_{22}^{-1} A_{21}\right) \rho_{1}+\left(N_{11}-N_{12} A_{22}^{-1} A_{21}\right) \rho_{1} u \\
y & =\left(C_{1}-C_{2} A_{22}^{-1} A_{21}\right) \rho_{1} .
\end{aligned}
$$

Averaged dynamics. Notice that $\epsilon$ in equation (4.6) is a fake parameter, i.e., it does not appear in the actual equations of motion. Nonetheless it marks where the 

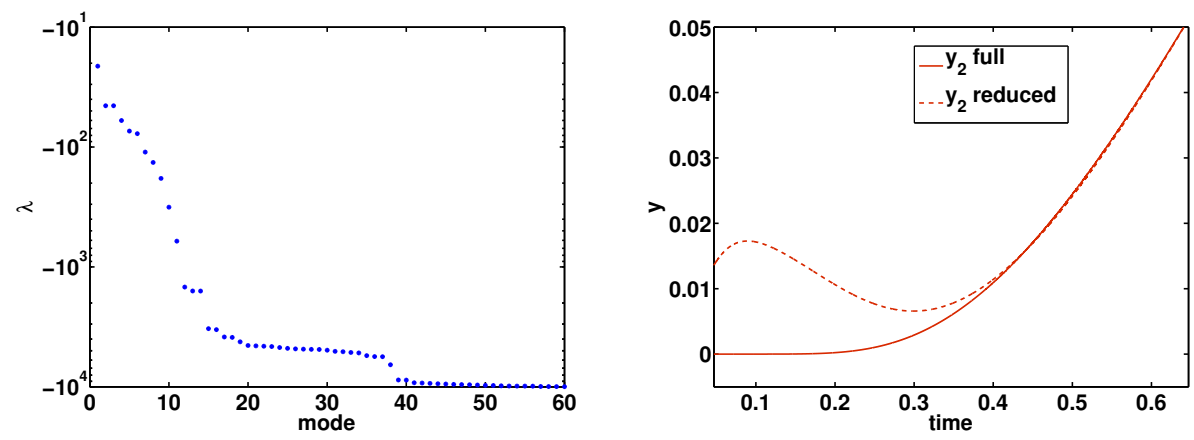

FiguRE 3. Contractivity of the invariant subspace: the left panel shows the largest 60 eigenvalues of the matrix $A_{22}$ that are responsible for the fast relaxation of the dynamics to the invariant subspace as the right plot shows (here $d=10$ ).

negligible HSVs enter the equations which is why we can expect (4.7) to yield a reasonable approximation whenever the negligible HSVs are small compared to the dominant ones.

By the $M$-matrix property of $A$ that is preserved under the balancing transformation $A \mapsto S(1) A T(1)$, the Schur complement $\bar{A}=A_{11}-A_{12} A_{22}^{-1} A_{21}$ is a singular $M$-matrix with a simple eigenvalue zero (see [8] and the references therein). ${ }^{2}$ As a consequence, the reduced system is positivity-preserving provided that the initial value remains positive upon balancing - which, unfortunately, is not always the case. The approximation result, however, guarantees that a positive output variables $y$ remains positive when the HSVs decay sufficiently fast. If the balanced variables stay positive, the vector $\bar{v}$ solving $\bar{A} \bar{v}=0$ can moreover be interpreted as the marginal equilibrium distribution of the dominant variables (again a simple computation that exploits the fact that $\bar{A}$ is the Schur complement of $A_{22}$ in $A$ ).

For a comparison of (4.5) and (4.7) we consider the following scenario: we discretize the Fokker-Planck equation (4.2) on the domain $I=[-2,2]$ using $n=400$ grid points. As initial value we choose $\rho_{0}(x) \propto \chi_{L}(x)$ where $\chi_{L}$ is the characteristic function of the set $L \subset \mathbb{R}$ and $L=[-1.2,-0.8]$ is symmetric around the left potential minimum at $x=-1$. The forcing $u$ is given by the mollified step function

$$
u(t)=\frac{1}{2}(\tanh (t-\pi)-1), \quad t \geq 0,
$$

that goes to zero as $t$ grows large. For the time-discretization we use a simple forward Euler scheme with constant step size $\Delta t=5 \cdot 10^{-5}$. The temperature was set to $\vartheta=1 / 2$. Since the matrix $A$ in our Fokker-Planck example violates the stability requirement, Assumption 1 on page 7 , the balancing transformation was computed by first doing a state transformation to the zero state and then stabilizing $A$ by shifting $A \mapsto A-s I$ with $s=10^{-3}$ and scaling the control input according to $u \mapsto 2 u$ (see Appendix A below). The dominant HSVs and the 400dimensional reference trajectory together with its approximant of dimension $d=10$ are shown in Figure 2. The blue curve is the probability in the left well whereas the red curve depicts how the right well is populated; observe that the population maximum is reached about $t \approx \pi$ slightly before the control is turned down. Once the control is switched off, the populations of left and right well start approaching their equilibrium values.

\footnotetext{
${ }^{2}$ Note that the $M$-matrix property does not need to be preserved under the scaled balancing transformation $A \mapsto S(\epsilon) A T(\epsilon)$ which is not a similarity transformation.
} 

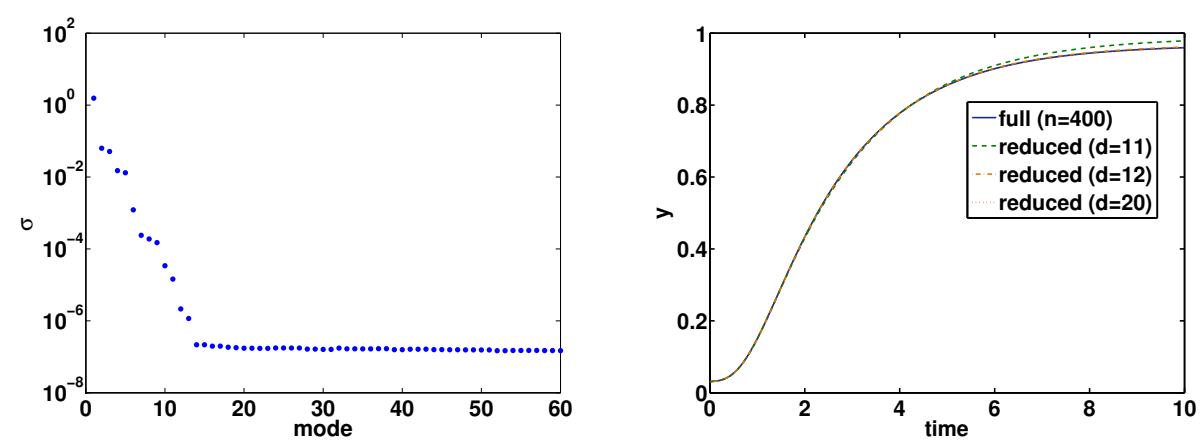

Figure 4 . Skew potential $W_{\mathrm{s}}$ : HSVs (the first 60 are shown) and the time evolution of the population of the right well for various approximants $(d=11,12,20)$.

Note that the initial values in the right panel of Figure 2 have not been projected to the invariant subspace; in point of fact starting from the invariant subspace would require that $u(0)=0$. But if the initial values do not lie in the invariant subspace of the fast dynamics then the dynamics have to relax to the invariant subspace before the limiting dynamics kicks in (see also the discussion below). The relaxation to the invariant subspace is demonstrated in the right panel of Figure 3. It can be seen that for sufficiently small HSVs (i.e., small enough $\epsilon$ ) the relaxation occurs quickly; notice that the fast dynamics relaxes even though the control force is still of order 1. The latter is due to the fact that the invariant subspace is contractive whenever $u$ is not too large (compare Section 3.2). The contraction condition is further justified by noting all the eigenvalues of $A_{22}$ in equation (3.4) are strictly negative, with a spectral gap of about $\delta \approx 3$ (see left panel of Fig. 3 ).

4.2. Non-decaying control force and positivity. The second example is to illustrate some of the subtleties and pitfalls of the method. To this end we consider a skew double well potential defined by

$$
W_{\mathrm{s}}(x)=\left(x^{2}-1\right)^{2}+x .
$$

In comparison with the symmetric potential, the left potential well is lowered and therefore carries the overall statistical weight if the system is in equilibrium.

Suppose that initially at $t=0$ the system is in the stationary state $\rho_{0}$ given by the eigenvector $\rho_{0}$ of $A$ to the simple eigenvalue zero. Apart from the modified potential, the discretization is essentially the same as before with $I=[-2,2], n=400$, $\vartheta=1 / 2$ and zero-flux boundary conditions. In contrast to the previous example and in violation of the theoretical assumptions that underlie the averaging result we choose an external force that is non-decaying. More precisely we set

$$
u(t)=2(1-\exp (-2 t)), \quad t \geq 0,
$$

which is clearly not square integrable over the positive real line. By the choice of $u$, the effective potential $V(x, u)=W_{\mathrm{s}}(x)-u x$ is turned into $W_{\mathrm{s}}^{-}=\left(x^{2}-1\right)^{2}-x$ as $t \rightarrow \infty$, which is exactly the reverse of $W_{\mathrm{s}}$ with the right well lowered.

As observable we choose the population of the right potential well, i.e., $C=P_{R}$ with $P_{R}$ denoting again the discrete characteristic function of the right well. The initial populations in the left and right wells are $\rho_{0, L}=0.9685$ and $\rho_{0, R}=0.0315$. We integrate the system up to the final time $T=10$ at which $W(x, u) \approx W_{\mathrm{s}}^{-}(x)$ and the population are $\rho_{L}=0.0404$ and $\rho_{R}=0.9596$.

As before, balancing and averaging is performed after a change of variables $\rho \mapsto$ $\rho+\rho_{\infty}$ and shifting $A \mapsto A-s I$ with $s=10^{-3}$ with an additional scaling $u \mapsto 2 u$ 

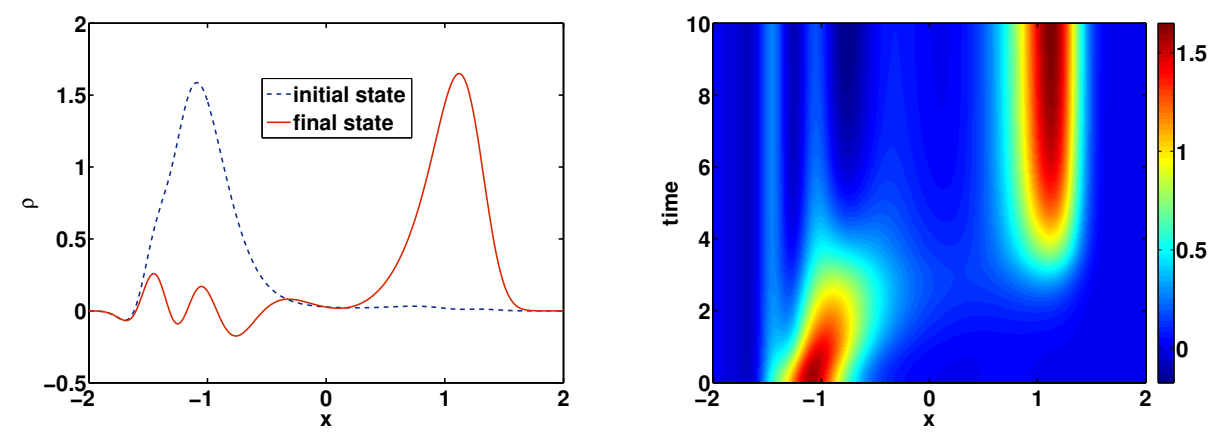

Figure 5. Negative densities: the left panel shows the spatial density distribution for $t=0$ and $t=10$ (final state) for an approximant of order $d=12$ (cf. left panel of Figure 4). The complete spatio-temporal density evolution is depicted in the right panel.

of the control input. ${ }^{3}$ Singular values and the time evolution of the observable $y=P_{R} \rho$ for various orders of approximation, $d$, are shown in Figure 4. Apparently the dynamics are well reproduced for $d \geq 12$ (see right panel of the figure).

But as usual the devil is in the details: Closer inspection reveals that the state variables other than the observables do not remain positive when $d$ is too small. Figure 5 shows an instance for $d=12$; it can be seen that both initial and final state $\rho(0)$ and $\rho(T)$ assume negative values in regions of low density (here: in the left well). The negative outliers disappear for $d \geq 20$. This behaviour is well in accordance with the limit result: Firstly, the $M$-matrix property of $A$ or $\bar{A}$, respectively, preserves positivity of the solution provided that the initial values are positive. This however does not need to be the case, because the balanced and truncated initial value is not necessarily positive. Secondly, the limit result essentially asserts that the approximation error is of the order of the negligible HSVs, so it does not come as a surprise that negative values appear in regions of low density; as $d$ is increased the outliers should vanish which is in agreement with the numerical findings. Thirdly, the projection to the invariant subspace may produce initial values that are no longer nonnegative; the same is true for the observables although the balancing transformation itself leaves the output variable invariant.

Remark 4.1. For the sake of completeness we computed also the truncated version of (4.5), i.e., the reduced system that is obtained from (4.6) by setting $\rho_{2}=0$ and compared the solutions to the averaged ones. In terms of the output variables y both methods yielded almost equally accurate approximants (cf. the recent work $[7,60]$ ). However, other than the Schur complement, truncation does not preserve the $M$ matrix property with the simple eigenvalue zero. As a consequence, the truncated system may not admit a stationary distribution. Moreover, the low lying eigenvalues of the Fokker-Planck operator that describe slow relaxation processes in the system-recall that the first nonzero eigenvalue can be used to estimate the convergence towards the stationary distribution - are well approximated by the singular perturbation method, but not so by the truncated system. The latter should not come as a surprise as the singular perturbation approach specifically aims at approximating the slow variables of the system. We leave the discussion of the approximation of the spectral properties of a diffusion process by reduced order models to future work.

\footnotetext{
${ }^{3}$ Alternatively, one may consider to split off the stationary state and balance the equations only on the stable orthogonal complement. However in our case, the results are found not to depend on this choice.
} 


\section{ACKNOWLEDGEMENT}

The authors thank the DFG Research Center Matheon "Mathematics for Key Technologies" (FZT86) and the DFG Collaborative Research Centre SFB 450 in Berlin for financial support of their work. Carsten Hartmann extends his thanks to John Maddocks for hosting him at the École Polytechnique Fédérale de Lausanne (EPFL) where a good deal of this article was written. We gratefully acknowledge the constructive comments from the anonymous referees and the Associate Editor.

\section{Appendix A. Generalized Lyapunov equations}

Consider the generalized Lyapunov equation

$$
A W_{c}+W_{c} A^{*}+\sum_{k=1}^{m} N_{k} W_{c} N_{k}^{*}+B B^{*}=0,
$$

and recall that if $A$ is Hurwitz there are constants $\lambda, \mu>0$, such that $\|\exp (A t)\| \leq$ $\lambda \exp (-\mu t)$ where $\|\cdot\|$ is any suitable matrix norm. If moreover

$$
\frac{\lambda^{2}}{2 \mu} \sum_{k=1}^{m}\left\|N_{k}\right\|^{2}<1
$$

the generalized (controllability) Gramian $W_{c}$ exists [32]. If the pair $(A, B)$ is completely controllable, i.e., if $\operatorname{rank}\left(B A B A^{2} B \ldots A^{n-1} B\right)=n$, then $W_{c}$ is positive definite [62].

Since direct methods for solving generalized Lyapunov equations have a numerical complexity $\mathcal{O}\left(n^{6}\right)$ computing Gramians is challenging even for medium-sized systems. For $A$ Hurwitz, the obvious iterative scheme is (see, e.g., [66])

$$
A X_{j+1}+X_{j+1} A^{*}=-\sum_{k=1}^{m} N_{k} X_{j} N_{k}^{*}-B B^{*}, \quad X_{0}=0
$$

which requires the solution of a standard Lyapunov equation in each step. Convergence $X_{j} \rightarrow W_{c}$ is guaranteed by the following result that is due to Damm $[17]$.

Lemma A.1. Let the linear operator $\mathcal{L}_{A}: \mathbb{R}^{n \times n} \rightarrow \mathbb{R}^{n \times n}$ be defined by $\mathcal{L}_{A}(X)=$ $A X+X A^{*}$ and let $\Pi: \mathbb{R}^{n \times n} \rightarrow \mathbb{R}^{n \times n}$ be nonnegative in the sense that $\Pi(X) \geqslant 0$ for $X \geqslant 0$. Further assume that the spectrum of $\mathcal{L}_{A}$ is contained in the closed left half-plane (including the imaginary axis) and that for a given $Y \geqslant 0$ there exists a nonnegative definite matrix $\hat{X} \geqslant 0$ such that $\left(\mathcal{L}_{A}+\Pi\right) \hat{X} \leqslant-Y$. Then there exists a minimal nonnegative definite solution $X_{-}$satisfying

(1) $X_{-} \geqslant 0$ and $\left(\mathcal{L}_{A}+\Pi\right) X_{-}=-Y$

(2) If $\hat{X} \geqslant 0$ such that $\left(\mathcal{L}_{A}+\Pi\right) \hat{X} \leqslant-Y$, then $\hat{X} \geqslant X_{-}$.

(3) $X_{j} \rightarrow X_{-}$, where $X_{j}$ for $j=0,1,2, \ldots$ is defined via

$$
X_{j+1}=-\mathcal{L}_{A-s I}^{-1}\left(\Pi\left(X_{j}\right)+2 s X_{j}+Y\right), \quad X_{0}=0, \quad s>0 .
$$

Setting $\Pi(X)=\sum_{k=1}^{m} N_{k} X N_{k}^{*}$ and $s=0$ Lemma A.1 implies that (A.2) converges to $W_{c}$ if the solvability condition (A.1) is met (cf. [7]).

Unstable systems I. In both of the previous numerical examples the matrix $A$ has a simple eigenvalue zero and $B=0$. However we may exploit the fact that $A$ has a nontrivial kernel and transform the homogeneous equation

$$
\dot{\rho}=A \rho+\sum_{k=1}^{m} N_{k} \rho u_{k}, \quad \rho(0)=\rho_{0},
$$


by doing a change of variables $\rho \mapsto \rho+v$ with $A v=0$. This yields

$$
\dot{\rho}=A \rho+\sum_{k=1}^{m} N_{k} \rho u_{k}+B u, \quad \rho(0)=\rho_{0}+v,
$$

with $B=-\left(N_{1} v, \ldots, N_{m} v\right)$. The difference between the homogeneous and the inhomogeneous system is that the latter has $\rho=v$ as stationary state whereas the other one has the stationary state $\rho=0$.

Clearly the Gramians do not exist if $A$ is not Hurwitz. In accordance with Lemma A.1 we may enforce stability by shifting the matrix $A$ according to $A \mapsto A-s I$ for a suitable $s>0$; cf. also [63] for the use of shifting for linear system. Physically, shifting amounts to a constant killing rate $s$ in the Fokker-Planck equation and makes the zero state $\rho=0$ the unique asymptotically stable fixed point. Alternatively one may split off the stationary state $v=\rho_{\infty}$ and balance only the orthogonal complement in which the dynamics are asymptotically stable. This approach has the advantage that it preserves the stationary state and that the computed Gramians are the Gramians associated with the true dynamics (i.e., without shifting). In both of our examples neither method turned out to be better than the other in terms of approximation quality of the reduced systems.

Unstable systems II. Now suppose that (A.1) does not hold while $A$ is Hurwitz. In this case we may replace the control by the scaled control $u \mapsto u / \mu$ for $0<\mu<1$. Invariance of (2.1) or (4.5) then requires that the coefficients scale according to $B \mapsto \mu B$ and $N_{k} \mapsto \mu N_{k}$ whereupon the system is altered according to

$$
\begin{aligned}
& \dot{\rho}=A \rho+\sum_{k=1}^{m}\left(\mu N_{k}\right) \rho \frac{u_{k}}{\mu}+(\mu B) \frac{u}{\mu}, \quad \rho(0)=\rho_{0} \\
& y=C \rho,
\end{aligned}
$$

and the generalized Lyapunov equation has to be replaced by (cf. [14])

$$
A W_{c}+W_{c} A^{*}+\mu^{2} \sum_{k=1}^{m} N_{k} W_{c} N_{k}^{*}+\mu^{2} B B^{*}=0
$$

The scaling as such clearly changes the Gramian $W_{c}$. Roughly speaking, we expect that the HSVs decay faster as $\mu$ becomes small (for $\mu \rightarrow 0$ the system becomes completely uncontrollable). But since the nullspace of $W_{c}$ is not affected by the scaling we still expect that, to lowest order, the ordering of the HSVs is not changed as long as $\mu$ is close to one.

\section{Appendix B. Sampling the generalized Gramians}

Instead of solving the generalized Lyapunov equation (2.6) directly, we may compute $W_{c}$ as the covariance matrix of a continuous-time Markov process that is governed by the following stochastic differential equation

$$
d X_{t}=A X_{t} d t+\sum_{k=1}^{m} N_{k} X_{t} d W_{k, t}+B d W_{t}, \quad X_{0}=0 .
$$

that is the stochastic analog of the deterministic control system

$$
\dot{x}=A x+\sum_{k=1}^{m} N_{k} x u_{k}+B u, \quad x(0)=0 .
$$

Here $W_{t}$ denotes standard Brownian motion in $\mathbb{R}^{m}$. To see this, it is helpful to note that $\mathbf{E} X_{t}=0$. By Itô's formula [51], it follows that

$$
d\left(X_{t} X_{t}^{*}\right)=X_{t} d X_{t}^{*}+d X_{t} X_{t}^{*}+\sum_{k=1}^{m}\left(N_{k} X_{t}+b_{k}\right)\left(N_{k} X_{t}+b_{k}\right)^{*}
$$


with the $b_{k} \in \mathbb{R}^{n}$ denoting the columns of the matrix $B$, i.e., $B=\left(b_{1}, \ldots, b_{m}\right)$. Now set $S_{t}=\mathbf{E} X_{t} X_{t}^{*}$. Inserting the differential equation for $d X_{t}$, taking the expectation, and interchanging expectation and differentiation, it follows that

$$
\dot{S}_{t}=A S_{t}+S_{t} A^{*}+\sum_{k=1}^{m} N_{k} S_{t} N_{k}^{*}+B B^{*} .
$$

Recall that the existence of the Gramian $W_{c}$ in (2.6) followed from (A.1). Equivalently the solvability condition (A.1) guarantees that the solutions of (B.1) are mean-square stable, i.e., for $B=0$, we have $\mathbf{E}\left|X_{t}\right|^{2} \rightarrow 0$ as $t \rightarrow \infty$ (see, e.g., [18] and the references therein). For $B \neq 0$ it therefore follows that $\dot{S}_{t} \rightarrow 0$ which entails

$$
W_{c}=\lim _{t \rightarrow \infty} S_{t} .
$$

The observability Gramian can be sampled in an analogous fashion (cf. [36]).

\section{REFERENCES}

[1] S.A. Al-Baiyat and M. Bettayeb. A new model reduction scheme for k-power bilinear systems. In: Proc. 32nd IEEE Conf. Decis. Control, pages 22-27, 1993.

[2] A.C. Antoulas. Approximation of Large-Scaled Dynamical Systems. SIAM, 2005.

[3] Z. Artstein. Invariant measures of differential inclusions applied to singular perturbations. Weizman Institute Research Report, 1998.

[4] Z. Bai and D. Skoogh. A projection method for model reduction of bilinear dynamical systems. Linear Algebra Appl. 415:406-425, 2006.

[5] D. Bakry, M. Emery. Hypercontractivité des semi-groupes de diffusion. C.R. Acad. Sci. Paris I 299:775-778: 1984.

[6] P. Benner, J.-R. Li, and T. Penzl. Numerical solution of large-scale Lyapunov equations, Riccati equations, and linear-quadratic optimal control problems. Numer. Linear Algebra Appl. 15:755-777, 2008.

[7] P. Benner, T. Damm. Lyapunov equations, energy functionals, and model order reduction. SIAM J. Control Optim. 49:686-711, 2011.

[8] A. Berman and R.J. Plemmons. Nonnegative Matrices in the Mathematical Sciences, Academic Press, 1979.

[9] T. Breiten and P. Benner. Interpolation-Based $\mathcal{H}_{2}$-Model Reduction of Bilinear Control System. SIAM J. Matrix Anal. Appl. 33:859-885, 2012.

[10] T. Breiten and T. Damm. Krylov subspace methods for model order reduction of bilinear control systems. Syst. Control Lett. 59, 443-450, 2011.

[11] N. Bogolyubov and Y. Mitropolskii. Asymptotic methods in the theory of nonlinear oscillations. Gordon and Breach, 1961.

[12] T. Breiten and T. Damm. Krylov subspace methods for model order reduction of bilinear control systems. Systems \& Control Letters 59:443-450, 2010.

[13] M. Condon and R. Ivanov. Empirical balanced truncation for nonlinear systems. J. Nonlinear Sci. 14:405-414, 2004.

[14] M. Condon and R. Ivanov. Nonlinear systems - algebraic gramians and model reduction. COMPEL 24:202-219, 2005.

[15] M. Condon and R. Ivanov. Krylov subspace from bilinear representations of nonlinear systems. COMPEL 26:11-26, 2007.

[16] P. D'Alessandro, A. Isidori, and A. Ruberti. Realization and structure theory of bilinear dynamical systems. SIAM J. Cont. 12:517-535, 1974.

[17] T. Damm, Direct methods and ADI-preconditioned Krylov subspace methods for generalized Lyapunov equations. Numer. Linear Algebra Appl. 15:853-871, 2008.

[18] T. Damm. Rational Matrix Equations in Stochastic Control, LNCIS \# 297, Springer, 2004.

[19] S. Djennoune and M. Bettayeb. On the structure of energy functions of singularly perturbed bilinear systems. J. Robust Nonlinear Control 15:601-618, 2005.

[20] A. Dontchev, T. Dontchev, and I. Slavov. A Tichonov-type theorem for singularly perturbed differential inclusion. Nonlinear Analysis 26:1547-1554:1996.

[21] L. Feng and P. Benner. A note on projection techniques for model order reduction of bilinear systems. AIP Conf. Proc. - International Conference of Numerical Analysis and Applied Mathematics 936:208-211, 2007.

[22] K. Fernando and H. Nicholson. Singular perturbational model reduction of balanced systems. IEEE Trans. Automat. Control AC-27:466-468, 1982.

[23] G.M. Flagg. Interpolation Methods for the Model Reduction of Bilinear Systems. PhD Thesis, Virginia Tech, 2012. 
[24] E.L. Florin, V.T. Moy, and H.E. Gaub. Adhesion forces between individual ligand-receptor pairs. Science 264:415-417, 1994.

[25] M.I. Freidlin and A.D. Wentzell. Random Perturbations of Dynamical Systems. Springer, 1998.

[26] K. Fujimoto and J.M.A. Scherpen. Singular value analysis and balanced realizations for nonlinear systems. In: Model Order Reduction: Theory, Research Aspects and Applications, W.H.A. Schilders, H.A. Vorst, J. van der Rommes (eds.), Berlin, Heidelberg, pp. 251-272, 2008.

[27] K. Fujimoto and J.M.A. Scherpen. Balanced realization and model order reduction for nonlinear systems based on singular value analysis. SIAM J. Control Optim. 48:4591-4623, 2010.

[28] V. Gaitsgory. Suboptimization of singularly perturbed control systems. SIAM J. Control Optim. 30:1228-1249, 1992.

[29] Z. Gajic and M. Lelic. Singular perturbation analysis of system order reduction via system balancing. Proc. Amer. Control Conf. 4:2420-2424, 2000.

[30] K. Glover. All optimal Hankel-norm approximations of linear multivariable systems and their $L^{\infty}$-error bounds. Int. J. Control 39:1115-1193, 1984.

[31] G. Grammel. Averaging of singularly perturbed systems. Nonlinear Analysis 28:1851-1865, 1997.

[32] W.S. Gray and J. Mesko. Energy functions and algebraic Gramians for bilinear systems. In: Preprints of the 4th IFAC Nonlinear Control Systems Design Symposium, Enschede, Netherlands, pp. 103-108, 1998.

[33] W.S. Gray and J. Mesko. Observability functions for linear and nonlinear systems. Systems E Control Letters 38:99-113, 1999.

[34] W.S. Gray and J.M.A. Scherpen. On the nonuniqueness of singular value functions and balanced nonlinear realizations. Systems \& Control Letters 44:219-232, 2001.

[35] C. Hartmann, V.-M. Vulcanov, and Ch. Schütte. Balanced truncation of linear second-order systems: a Hamiltonian approach. Multiscale Model. Simul. 8:1348-1367, 2010.

[36] C. Hartmann. Balanced model reduction of partially-observed Langevin equations: an averaging principle. Math. Comp. Model. Dyn. 17:463-490, 2011.

[37] P. Holmes, J.L. Lumley, and G. Berkooz. Turbulence, Coherent Structures, Dynamical Systems and Symmetry. Cambridge University Press, 1996.

[38] A. Isidori and A. Ruberti. Realization theory of bilinear systems. In: Geometric Methods in System Theory, D.Q. Mayne and R.W. Brocken (eds.), Dordrecht, pp. 81-130, 1973.

[39] M.S.Z. Kellermayer, S.B. Smith, H.L. Granzier, and C. Bustamante. Folding-unfolding transitions in single Titin molecules characterized with laser tweezers. Science 276:1112-1116, 1997.

[40] R.Z. Khas'minskii. A limit theorem for the solution of differential equations with random right-hand sides. Theory Prob. Applications, 11:390-406, 1966.

[41] E. Klipp, R. Herg, A. Kowald, C. Wierling, and H. Lehrach. Systems Biology in Practice: Concepts, Implementation and Application. Wiley-VCH, 2005.

[42] P.V. Kokotovic. Applications of singular perturbation techniques to control problems. SIAM Review 26:501-550, 1984.

[43] H.J. Kushner and P. Dupuis. Numerical Methods for Stochastic Control Problems in Continuous time. Springer, 2001.

[44] S. Lall, J.E. Marsden, and S. Glavaški. A subspace approach to balanced truncation for model reduction of nonlinear control systems. Int. J. Robust Nonlinear Control 12:519-535, 2002

[45] Y.Q. Lin, L. Bao, and Y. Wei. A model-order reduction method based on Krylov subspaces for mimo bilinear dynamical systems. J. Appl. Math. Comput. 25:293-304, 2007.

[46] Y.Q. Lin, L. Bao, and Y. Wei. Order reduction of bilinear MIMO dynamical systems using new block Krylov subspaces. Comput. Math. Appl. 58:1093-1102, 2009.

[47] Y. Liu and B.D.O. Anderson. Singular perturbation approximation of balanced systems. Int. J. Control 50, pp. 1379-1405, 1989.

[48] H. Mabuchi and N. Khaneia. Principles and applications of control in quantum systems. J. Robust Nonlinear Control 15:647-667, 2005.

[49] R. Merkel, P. Nassoy, A. Leung, K. Ritchie, and E. Evans. Energy landscapes of receptorligand bonds explored with dynamic force spectroscopy. Nature 397:50-53, 1999.

[50] B.C. Moore. Principal component analysis in linear systems: controllability, observability, and model reduction. IEEE Trans. Automat. Control AC-26:17-32, 1981.

[51] B. Øksendal. Stochastic Differential Equations: An Introduction with Applications. Springer, 2003.

[52] G.C. Papanicolaou. Some probabilistic problems and methods in singular perturbations. Rocky Mountain J. Math. 6:653-674, 1976.

[53] G.A. Pavliotis and A.M. Stuart. Multiscale Methods: Averaging and Homogenization. Springer, 2008. 
[54] J.R. Phillips. Projection-based approaches for model reduction of weakly nonlinear, timevarying systems. IEEE T. Comput. Aided. D. 22:171-187, 2003.

[55] A. Quarteroni and A. Valli. Domain Decomposition Methods for Partial Differential Equations. Oxford Science Publications 1999.

[56] M. Rief, M. Gautel, F. Oesterhelt, J.M. Fernandez, and H.E. Gaub. Reversible unfolding of individual Titin Immunoglobulin domains by AFM. Science 276:1109-1112, 1997.

[57] H. Risken. The Fokker-Planck Equation: Methods of Solutions and Applications. Springer, 1996.

[58] C.W. Rowley. Model reduction for fluids using balanced proper orthogonal decomposition. Int. J. Bifurcation Chaos Appl. Sci. Eng. 15:997-1013, 2005.

[59] R. Samar, I. Postlethwaite and Da-Wei Gu. Applications of the singular perturbation approximation of balanced systems. Proc. IEEE Conf. Control Appl. 3:1823-1828, 1994.

[60] B. Schäfer-Bung, C. Hartmann, B. Schmidt and Ch. Schütte. Dimension reduction by balanced truncation: Application to light-induced control of open quantum systems. J. Chem. Phys. 135:, 014112, 2011.

[61] J.M.A. Scherpen. Balancing for nonlinear systems. Systems \& Control Letters 21:143-153, 1993.

[62] H. Schneider. Positive operators and an inertia theorem. Numer. Math. 7:11-17, 1965.

[63] M. Sznaier, A. Doherty, M. Barahona, H. Mabuchi, and J.C. Doyle. A new bound of the $L^{2}[0, T]$-induced norm and applications to model reduction. Proc. 2002 American Control Conference, pp. 1180-1185, 2002.

[64] A. Vigodner. Limits of singularly perturbed control problems with statistical dynamics of fast motions. SIAM J. Control Optim. 35:1-28, 1997.

[65] C. Villani. Topics in Mass Transportation. Graduate studies in Mathematics, AMS, 2003.

[66] E. Wachspress. Iterative solution of the Lyapunov matrix equation. Appl. Math. Lett. 1:87-90, 1988.

[67] X. Wang and Y. Jiang. Model reduction of bilinear systems based on Laguerre series expansion. J. Franklin Inst. 349:1231-1246, 2012.

[68] F. Watbled. On singular perturbations for differential inclusions on the infinite time interval. J. Math. Anal. Appl. 310:362-378, 2005.

[69] L. Zhang and J. Lam. On $\mathrm{H}_{2}$ model reduction of bilinear systems. Automatica 38:205-216, 2002.

[70] K. Zhou, J.C. Doyle and K. Glover. Robust and Optimal Control. Prentice Hall, New Jersey, 1998.

Institut für Mathematik, Freie Universität Berlin, 14195 Berlin, Germany

E-mail address: chartman@mi.fu-berlin.de

Institut für Mathematik, Freie Universität Berlin, 14195 Berlin, Germany

E-mail address: boris.schaefer-bung@fu-berlin.de

Institut für Mathematik, Technische Universität Berlin, 10623 Berlin, Germany

E-mail address: zueva@math.tu-berlin.de 